\title{
EFFICIENT ALGORITHM FOR GLOBALLY OPTIMAL UNEVEN ERASURE-PROTECTED PACKETIZATION OF SCALABLE CODE STREAMS
}

\author{
Sorina Dumitrescu, Xiaolin Wu and Zhe Wang \\ Dept. of Electrical and Computer Engineering \\ McMaster University, Hamilton, ON, Canada L8S 4K1 \\ sorina/xwu/zwang@mail.ece.mcmaster.ca
}

\begin{abstract}
A new algorithm is presented for rate-fidelity optimal packetization of scalable source bit streams with uneven erasure protection. It provides the globally optimal solution for input sources of convex rate-fidelity function and for a wide class of erasure channels, including channels for which the probability of losing $n$ packets is monotonically decreasing in $n$, and independent erasure channels with packet erasure rate smaller than 0.5. The time and space complexities of the new algorithm are both $O(N L)$, where $N$ is the number of packets and $L$ is the packet payload size, comparing to the $O\left(N L^{2}\right)$ time and space complexities of the existing globally optimal solution. When applied to SPIHT compressed images, the results of the proposed algorithm are virtually the same as the globally optima.
\end{abstract}

\section{INTRODUCTION}

In multimedia streaming over the Internet, the quality of service (QoS) henges on how well the problem of packet losses is dealt with. Optimal packetization of scalable source sequence with uneven erasure protection (UEP) offers a principled solution to the problem.

Scalable compression algorithms, such as SPIHT [5] and EBCOT [7], can reconstruct a coded signal to certain fidelity from any prefix of the code stream. This feature can be exploited by Reed-Solomon (RS) codes to generate a rate-distortion optimized UEP packetization. Specifically, a collection of (RS) block codes of the same length but decreasing redundancy are used to protect subsequent segments of the scalable source code stream, and forms the packets across the channel codewords. Any set of received packets can be used to reconstruct the source to some fidelity, and the fidelity increases in the number of received packets. We are interested in the problem of optimal UEP packetization under the criterion of maximizing the expected fidelity at the receiver, constrained by a given transmission budget.

Let $N$ be the number of packets to be transmitted, and $L$ the number of symbols in each packet (a symbol is a block of a fixed number of bits, usually 8 bits). In general, only a prefix of the scalable source sequence is packetized. This prefix of the source code stream is partitioned into $L$ consecutive segments, and each of these segments is protected by RS code. Let $m_{i}$ be the length (in symbols) of the $i$ th source segment, then the channel code assigned to protect it, is the $\left(N, m_{i}\right) \mathrm{RS}$ code. The stream of these $m_{i}$ source symbols followed by the $f_{i}=N-m_{i}$ redundancy symbols constitutes the $i$-th slice of the joint source-channel code. The packets are formed across the slices, i.e., the $n$ th packet contains the $n$-th symbol of each slice. The effect of the $\left(N, m_{i}\right)$ RS code applied to the $i$-th source segment is that, if at most $f_{i}$ of $N$ packets are lost, then all the $m_{i}$ source symbols of the $i$-th slice can be correctly recovered. However, since the scalable source sequence is only sequentially refinable, the $i$-th source segment can be decoded only if the previous $i-1$ segments are available. This requires that the number of redundancy symbols assigned to a slice be monotonically non-increasing in the slice index: $f_{1} \geq f_{2} \geq \cdots \geq f_{L}$, or equivalently, the number of source symbols allocated to each slice be monotonically non-decreasing in the slice index:

$$
m_{1} \leq m_{2} \leq \cdots \leq m_{L}
$$

Let $\mathbf{m}=\left(m_{1}, m_{2}, \cdots, m_{L}\right)$ be the vector whose components are the number of source symbols allocated to the slices. We call $\mathbf{m}$ the $L$-slice source allocation vector. Figure 1 illustrates the UEP packetization scheme.

Let $\phi(r)$ be the rate-fidelity function of the scalable source sequence, which is a monotonically non-decreasing function in rate $r \in\left[0, R_{\max }\right]$, where $r$ denotes the number of symbols in a prefix of the source sequence, and $R_{\max }$ is the total number of source symbols. Let $p_{N}(n)$, for $0 \leq$ $n \leq N$, denote the probability of losing $n$ packets out of $N$. The efficiency of the packetization scheme is measured by the expected fidelity of the reconstructed sequence at the decoder side, denoted by $\Phi(\mathbf{m})$. This quantity can be expressed as [3]

$$
\begin{gathered}
\Phi(\mathbf{m})=P_{N}(N) \phi(0)+\sum_{i=1}^{L} P_{N}\left(f_{i}\right)\left(\phi\left(r_{i}\right)-\phi\left(r_{i-1}\right)\right)= \\
P_{N}(N) \phi(0)+\sum_{i=1}^{L} P_{N}\left(N-m_{i}\right)\left(\phi\left(r_{i}\right)-\phi\left(r_{i-1}\right)\right),
\end{gathered}
$$




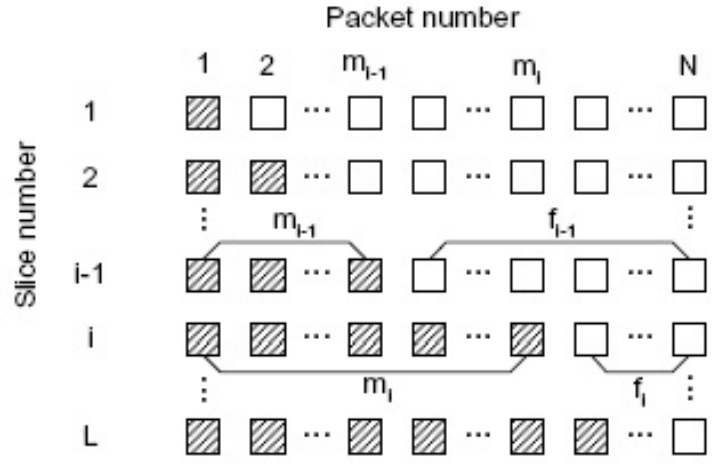

Fig. 1. Uni-group UEP packetization scheme. The slices are positioned horizontally and the packets vertically. The shaded squares represent the source symbols and the white squares represent redundancy symbols.

where $P_{N}(k)=\sum_{n=0}^{k} p_{N}(n), k=0,1, \cdots, N$, and $r_{i}=$ $\sum_{k=1}^{i} m_{k}, 1 \leq i \leq L, r_{0}=0$.

The objective of optimal UEP packetization under the rate-fidelity criterion is to find the $L$-slice source allocation vector $\mathbf{m}=\left(m_{1}, m_{2}, \cdots, m_{L}\right)$ that maximizes $\Phi(\mathbf{m})$, for given $N, L, p_{N}(n)$, and $\phi(r)$. Various algorithms have been proposed in the literature to find approximate or exact solutions. The approximation algorithms of $[3,4,6]$ assume the convexity of the rate-fidelity function, but cannot guarantee the global optimality of the solution even under this assumption. Among the globally optimal algorithms, the most efficient are those proposed in [2], namely a $O\left(N^{2} L^{2}\right)$ time complexity algorithm for the most general setting of the problem, and a $O\left(N L^{2}\right)$ running time algorithm for the case of convex rate-fidelity function and a wide class of erasure channels, including channels for which the probability function of losing $n$ packets is monotonically decreasing in $n$ and independent erasure channels with packet erasure rate no larger than $\frac{N}{2(N+1)}$.

The contribution of this work is a more efficient globally optimal algorithm for the latter case. The new algorithm is based on a Lagrangian formulation of the problem. For each value of the Lagrangian multiplier $\lambda$, the algorithm takes $O(N L)$ time. The number of iterations needed to find the optimal $\lambda$, and hence to complete the algorithm, is much smaller than $L$, leading to great savings of computations from the $O\left(N L^{2}\right)$ time algorithm of [2]. The memory usage also drops from $O\left(N L^{2}\right)$ in [2] to $O(N L)$. The saving in memory partially accounts for the increased speed of the new algorithm.

\section{NEW MODELING OF THE PROBLEM}

We assume that the rate-fidelity function is convex (more precisely, upward convex) and that $p_{N}(n)$ is decreasing in $n$ (the other case when the channel is an independent packet erasure channel will be discussed at the end of this section). It was shown in [2] that in this case optimal UEP packetization can be computed by maximizing the expression (2) without imposing the constraint (1). The first step in our development is to show that maximizing (2) is equivalent to solving a maximum-weight path problem constrained on the number of edges.

Consider the weighted directed acyclic graph $G=(V, E)$, whose nodes are identified with nonnegative integer numbers between 0 and $M$, where $M=\min \left(R_{\max }, N L\right)$, i.e. $V=\left\{0,1,2, \cdots, R_{\max }\right\}$, and any two nodes $u, v$ such that $0<v-u \leq N$ are connected by an edge, hence the set of edges is $E=\{(u, v) \mid 0 \leq u<v \leq M, v-u \leq N\}$. The weight of an edge $(u, v)$ is defined to be $w(u, v)=$ $P_{N}(N-v+u)(\phi(v)-\phi(u))$. Let the source vertex of the graph be 0 and let the set of final vertices coincide with $V$.

A path in the graph is any sequence of nodes such that any two consecutive nodes are connected by an edge. The weight of the path is the sum of weights of edges connecting the consecutive nodes. Note that any $L$-slice source allocation vector $\mathbf{m}$, not necessarily satisfying the constraint (1), can be associated to a path of $L$ edges in the graph $G$, starting at the source node and ending at a final node, namely the path: $r_{0}, r_{1}, \cdots, r_{L}$. This correspondence is one to one. Moreover, the weight of the path equals the value $\Phi(\mathbf{m})-P_{N}(N) \phi(0)$. Consequently, maximizing $\Phi(\mathbf{m})$ is equivalent to maximizing the weight of the path. In other words, the problem of optimal UEP packetization is equivalent to the problem of finding the path of maximum weight among all the paths from the source to a final node, which have exactly $L$ edges (the maximum-weight $L$-edge path problem). The graph $G$ can be assumed to be complete, i.e., a graph where each ordered pair $(u, v)$ of vertices with $u<v$, forms an edge, by setting to $-\infty$ the weight of pairs $(u, v) \notin E$.

The following result is essential to our development. We skip its proof due to limited space. The proof follows the same idea as the proof of Proposition 2 in [2].

Proposition 1. The graph $G$ satisfies the so-called Monge property, i.e.

$$
w\left(u_{1}, v_{1}\right)+w\left(u_{2}, v_{2}\right) \geq w\left(u_{1}, v_{2}\right)+w\left(u_{2}, v_{1}\right),
$$

for all $u_{1}, u_{2}, v_{1}, v_{2}$ such that $r_{0} \leq u_{1}<u_{2}<v_{1}<v_{2} \leq$ $M$.

The next result states the convexity (more exactly, the upward convexity) of $\bar{W}(l)$ as a function of $l$, where $\bar{W}(l)$ denotes the weight of the maximum-weight $l$-edge path from 
the source to a final node, in $G$. Its proof is given in Appendix.

Proposition 2. The inequality

$$
2 \bar{W}(l) \geq \bar{W}(l-1)+\bar{W}(l+1)
$$

holds for all $l, 1 \leq l \leq M$.

The maximum-weight $L$-edge problem, that we need to solve, is a constrained optimization problem. The constraint is on the number of edges in the path. The convexity result proved above implies that the Lagrangian method can be used to solve this problem exactly.

Let $\mathcal{P}$ denote the set of all paths from the source node to any terminal node, in the graph $G$. For any path $P \in \mathcal{P}$ let $W(P)$ denote its weight and $L(P)$ its length (the number of its edges). Then the problem of maximum-weight $L$-edge path in $G$ can be formulated as

$$
\begin{aligned}
& \operatorname{maximize}_{P \in \mathcal{P}} W(P) \\
& \text { subject to } L(P)=L .
\end{aligned}
$$

The underlying Lagrangian is $J(\lambda, P)=W(P)+\lambda L(P)$, over all paths $P \in \mathcal{P}$ and all real values $\lambda$. For each real $\lambda$, let $P_{\lambda}$ denote the path which maximizes $J(\lambda, P)$ over all $P \in \mathcal{P}$, i.e.,

$$
P_{\lambda}=\max _{P \in \mathcal{P}} J(\lambda, P),
$$

The convexity of $\bar{W}(l)$ implies that there is some real value $\lambda_{0}$ such that $L\left(P_{\lambda_{0}}\right)=L$. Then $P_{\lambda_{0}}$ is the solution of the constrained problem (5). Moreover, $\bar{W}(L-1)-$ $\bar{W}(L) \leq \lambda_{0} \leq \bar{W}(L)-\bar{W}(L+1)$ (i.e., $-\lambda_{0}$ corresponds to the slope of the curve $\bar{W}(\cdot)$ in the point $(L, \bar{W}(L)))$. These statements follow either according to the theory of Lagrangian multipliers or by direct proof in the spirit of [1].

Therefore, the $L$-edge maximum-weight path can be found by solving (6) in conjunction with a search on $\lambda$ until the number of the edges on the path becomes exactly $L$. To this end we derive from $G$ a parameterized graph $G(\lambda)$ by adding $\lambda$ to the weight of each edge of $G$. In the resulting parameterized graph $G(\lambda)$ the maximization problem of (6) reduces to an unconstrained maximum-weight path problem. This is because $J(\lambda, P)$ equals the weight of the path $P$ in $G(\lambda)$.

It is easy to check that, since the graph $G$ satisfies the Monge property, then $G(\lambda)$ satisfies the Monge property, too. Therefore, the maximum-weight paths from the source to each node can be found in $O(|V|)=O(N L)$ time and space by using the algorithm proposed in [8]. Further, by computing the maximum of these paths, in no more than $O(N L)$ time, the maximum-weight path of the graph is found. Consequently, for each $\lambda$, the maximization of (6) is solved in $O(N L)$ time and space.

To find the optimal $\lambda_{0}$, we use bisection search, since the length of $P_{\lambda}$ is non-decreasing as the parameter $\lambda$ increases [1]. More exactly, a search interval for $\lambda,\left[\lambda_{\text {low }}, \lambda_{\text {high }}\right]$ is maintained at any time. At the beginning $\lambda_{\text {low }}=-\frac{\phi(N L)}{L}$ and $\lambda_{\text {high }}=0$. At the beginning of each iteration, the current value of $\lambda$ is set to $\left(\lambda_{\text {low }}+\lambda_{\text {high }}\right) / 2$. If $L\left(P_{\lambda}\right)=$ $L$ the algorithm stops. Otherwise, depending on whether $L\left(P_{\lambda}\right)<L$ or $L\left(P_{\lambda}\right)>L$, the search interval $\left[\lambda_{\text {low }}, \lambda_{\text {high }}\right]$ is updated to $\left[\lambda, \lambda_{\text {high }}\right]$ or $\left[\lambda_{\text {low }}, \lambda\right]$ respectively. This technique ensures that the search interval for $\lambda$ becomes smaller after each iteration. However, since the path lengths $L\left(P_{\lambda}\right)$ take values only in a finite set, it follows that the interval $\left[L\left(P_{\lambda_{\text {low }}}\right), L\left(P_{\lambda_{\text {high }}}\right)\right]$ (which is guaranteed to include $L)$ may not change after some iterations. The first time it happens (but after the endpoints of the interval have both changed from their initial values), we switch to another strategy for updating $\lambda$, namely $\lambda=\left(\lambda_{\text {high }}-\lambda_{\text {low }}\right) /\left(L\left(P_{\lambda_{\text {low }}}\right)-\right.$ $\left.L\left(P_{\lambda_{\text {high }}}\right)\right)$. If after this switch, the interval of lengths $\left[L\left(P_{\lambda_{\text {low }}}\right), L\left(P_{\lambda_{\text {high }}}\right)\right]$ does not change after some iteration, then we stop concluding that the current $\lambda$ is the optimal one. This situation corresponds to the case when the function $\bar{W}(l)$ is linear for $l \in\left[L\left(P_{\lambda_{\text {low }}}\right), L\left(P_{\lambda_{\text {high }}}\right)\right]$. The desired $L$-edge path is constructed then from $P_{\lambda_{\text {low }}}$ and $P_{\lambda_{\text {high }}}$ in $O(L)$ time, in a similar way as that described in the proof of Proposition 2.

If $\alpha$ denotes the number of iterations until the optimal $\lambda$ is found, then the optimal UEP packetization can be solved in $O(\alpha N L)$ time, for channels with decreasing $p_{N}(n)$ and for convex rate-fidelity curves. We have empirically found that $\alpha$ increases extremely slow with $L$, thus supporting our claim of higher efficiency versus the $O\left(N L^{2}\right)$ time algorithm of [2]. The space complexity of the new algorithm is clearly linear in the transmission budget, i.e. $O(N L)$, because the current path does not have to be stored from one iteration to the next.

Assume now that the channel is an independent erasure channels with packet erasure rate no larger than $\frac{N}{2(N+1)}$. Let $n_{0}=\lfloor\epsilon(N+1)\rfloor$. It was proven in [2] that an optimal $L$-slice source allocation vector $\mathbf{m}$ exists such that $m_{i} \leq$ $N-n_{0}$ for all $i$. Then the graph $G$ is constructed such that only edges $(u, v)$ with $v-u \leq N-n_{0}$, to have finite weight (defined as previously), and all the other edges to have the weight $-\infty$. It was also shown in [2] that $p_{N}(n)$ is nonincreasing for $n \geq n_{0}$, which is the crucial ingredient to show that the modified graph satisfies the Monge property. Further, the same development applies as in the previous case.

\section{EXPERIMENTAL RESULTS}

We have tested the new algorithm on four images compressed by SPIHT [5]. The images and their sizes are: barb (576x720), boat $(576 \times 720)$, lena $(512 \times 512)$ and zelda $(512 \times 512)$. The fidelity measure used is PSNR. In order to have exact convexity of the rate-fidelity curve, we approximated the real PSNR curve by its upper convex hull (the same approxima- 
tion was also used by our predecessors [3, 4, 6]).

In order to test the number of iterations $\alpha$, we considered different values for $L$ (from 50 to 200, in increments of 25) and different values of $N$ (from 50 to 200, in increments of 25). In our experiments we simulated packet erasure channels with exponentially decreasing $p_{N}(n)$ and different mean packet loss rates: $0.15,0.2,0.25,0.3$. The number of iterations for all our tests ranges between 2 and 14 with an average of 9.61. The extreme values 2 and 14 were statistical outliers. The average number of iterations exhibits a very slight increase with $L$ : from 8.3 for $L=50$, to 10.5 for $L=200$. But for practical considerations, we can treat $\alpha$ as a constant.

The proposed algorithm is globally optimal for convex rate-fidelity curves, but for real images it is an approximation. To assess the quality of this approximation we compared the new algorithm against the globally optimal algorithm of [2]. We performed tests on all four images given above assuming a packet erasure channel with exponentially decreasing $p_{N}(n)$ and 0.2 mean packet loss rate. We considered two values for $L: L=50,100$, and three values for $N: N=100,150,200$. The new algorithm achieved solution within $0.01 \mathrm{~dB}$ of the optimal one, in $61.5 \%$ of the total of 24 cases. For the remaining cases the difference in expected PSNR versus the optimum was uniformly distributed in the range $[0.01,0.05] \mathrm{dB}$.

\section{CONCLUSION}

We have proposed a new efficient algorithm for uneven erasure packetization of scalable source code streams. The algorithm finds the globally optimal packetization for scalable code streams of convex rate-fidelity function and a large class of erasure channels. The time and space complexities of the new algorithm are linear in $N$ and in $L$, representing a significant improvement over the previous $O\left(N L^{2}\right)$ algorithm. For real SPIHT-compressed images, the new algorithm obtains solutions extremely close to the globally optimum.

\section{Appendix}

Proof of Proposition 2. Note that a similar result was proved in [1] (Corollary 7) for a complete graph with Monge property, but only one terminal node. In our graph all nodes are terminal, therefore the result of [1] is not applicable, but in our proof we will use an intermediary result of [1] (Lemma 6). Consider the paths $P_{1}$ and $P_{2}$ the $(l-1)$-link maximum weight path, and the $(l+1)$-link maximum-weight path. Let $P_{1}$ be $r_{0}, r_{1}, \cdots, r_{l-1}$, and let $P_{2}$ be $r_{0}, r_{1}^{\prime}, \cdots, r_{l+1}^{\prime}$. The idea of the proof is to construct two $l$-link paths $Q_{1}$ and $Q_{2}$ such that the following relation to hold:

$$
W\left(Q_{1}\right)+W\left(Q_{2}\right) \geq W\left(P_{1}\right)+W\left(P_{2}\right)
$$

We need to distinguish between three cases.
Case 1: $r_{l+1}^{\prime} \leq r_{l-1}$. According to Lemma 6 in [1], a path $Q_{1}$ from the source to $r_{l-1}$, and a path $Q_{2}$ from the source node to $r_{l+1}^{\prime}$, each of $l$ edges, can be constructed such that (7) to hold.

Case 2: $r_{l}^{\prime} \leq r_{l-1}<r_{l+1}^{\prime}$. Construct the $l$-edge paths $Q_{1}$ from $r_{0}$ to $r_{l-1}$, and the $(l-1)$-edge path $Q_{2}^{\prime}$ from $r_{0}$ to $r_{l}^{\prime}$ as in Lemma 6 in [1], such that the sum of the weights of $Q_{1}$ and $Q_{2}^{\prime}$ is larger or equal than the weight of $P_{1}$ plus the weight of the sum of weights of the first $l$ edges of $P_{2}$ (i.e. the prefix of $P_{2}$ up to node $r_{l}^{\prime}$ ). Then construct $Q_{2}$ by appending the edge $\left(r_{l}^{\prime}, r_{l+1}^{\prime}\right)$ to $Q_{2}^{\prime}$. Clearly, (7) is now satisfied.

Case 3: $r_{l-1}<r_{l}^{\prime}$. Let $Q_{2}$ be the prefix of the path $P_{2}$ up to the node $r_{l}^{\prime}$. Denote $a=r_{l-1}+r_{l+1}^{\prime}-r_{l}^{\prime}$ and let $Q_{1}$ be the path obtained by appending the edge $\left(r_{l-1}, a\right)$ to $P_{1}$. Then, in order to prove (7) it is enough to show that $w\left(r_{l-1}, a\right) \geq w\left(r_{l}^{\prime}, r_{l+1}^{\prime}\right)$. This inequality is equivalent to $\phi(a)-\phi\left(r_{l-1}\right) \geq \phi\left(r_{l+1}^{\prime}\right)-\phi\left(r_{l}^{\prime}\right)$, which follows from the upward convexity of $\phi$.

\section{REFERENCES}

[1] A. Aggarwal, B. Schieber, and T. Tokuyama, "Finding a minimum-weight $k$-link path in graphs with the concave monge property and applications", Discrete \& Computational Geometry, vol. 12, pp. 263-280, 1994.

[2] S. Dumitrescu, X. Wu, and Z. Wang, "Globally optimal uneven error-protected packetization of scalable code streams", IEEE Trans. on Multimedia, vol. 6, no. 2, pp.230-239, April 2004.

[3] A. E. Mohr, R. E. Ladner, and E.A. Riskin, " Approximately optimal assignment for unequal loss protection", in Proc. ICIP 2000 International Conf. on Image Processing, vol. 1, pp. 367-370, Sept. 2000.

[4] R. Puri, and K. Ramchandran, "Multiple description source coding through forward error correction codes", Proc. 33rd Asilomar Conference on Signals, Systems, and Computers, vol. 1, pp. 342-346, Oct. 1999.

[5] A. Said and W. A. Pearlman, "A new, fast, and efficient image codec based on set partitioning in hierarchical trees", IEEE Trans. Circuits and Systems for Video Tech., vol. 6, pp. 243-250, June 1996.

[6] V. Stankovic, R. Hamzaoui, and Z. Xiong, ”Packet loss protection of embedded data with fast local search", Proc. ICIP'2002, vol. 2, pp. II-165-II-168, Sept. 2002.

[7] D. Taubman, "High performance scalable image compression with EBCOT," IEEE Trans. Image Processing, vol. 9, pp. 1158-1170, July 2000.

[8] R. Wilber, "The concave least-weight subsequence problem revisited", J. of Algorithms, 9(1988), pp. 418-425. 